\title{
ANÁLISIS
}

\section{WHISTLEBLOWING Y DATOS MASIVOS: MONITORIZACIÓN Y CUMPLIMIENTO DE LA ETICA Y LA RESPONSABILIDAD SOCIAL}

\author{
Whistleblowing \& big data: Monitoring and \\ compliance of ethics and social responsibility
}

\section{Patrici Calvo y Cristian Osal}

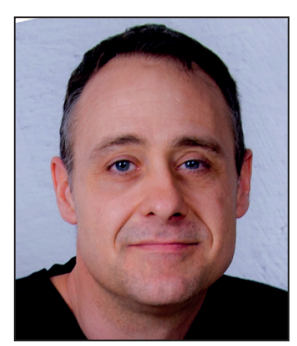

Patrici Calvo es licenciado en Humanidades por la Universitat Jaume I (UJI) (Premio extraordinario de carrera), master en Nuevas tendencias y procesos de innovación en comunicación por la UJI, master en Responsabilidad social corporativa por la Universidad Politécnica de Valencia, formador de formadores en responsabilidad social empresarial por la Universidad de Buenos Aires, y doctor en Filosofía Moral por la UJI. Es profesor de ética en el Departamento de Filosofía y Sociología de la UJl. Ha realizado diversas estancias de investigación, como en el Uehiro Centre for Practical Ethics de la University of Oxford (UK), entre otros centros.

https://orcid.org/0000-0002-3228-9019

calvop@uji.es

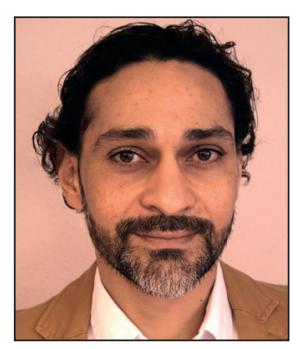

Cristian Osal es licenciado en Filosofía por la Universidad Católica Andrés Bello de Caracas (Venezuela) y master universitario en Ética y democracia por la Universitat Jaume I. Es investigador predoctoral, realizando un estudio sobre los aspectos éticos de los datos masivos (big data) y su potencialidad en el desarrollo de sistemas de monitorización de la ética y la responsabilidad social en la empresa.

https://orcid.org/0000-0003-0715-833X

al367828@uji.es

Universitat Jaume I, Facultad de Filosofía y Sociología Avda. Vicent Sos Baynat, s/n. 12071 Castellón de la Plana, España

\section{Resumen}

La revolución de los datos masivos (big data) supone un reto y una oportunidad para la empresa. En el ámbito de la ética y la responsabilidad social, los datos masivos y la cada vez más accesible y versátil tecnología de procesamiento de la información disponible, permite a la empresa generar nuevos canales de comunicación y recabar información precisa, detallada y continuamente actualizada de sus impactos económicos, sociales y medioambientales; de los intereses y expectativas legítimas en juego; de la confianza, reputación y afinidad que despierta entre sus stakeholders, de los afectos que producen sus acciones y decisiones, entre otras muchas cosas. El objetivo de este estudio es mostrar un sistema de monitorización y cumplimiento de la ética y la responsabilidad social que, basado en el whistleblowing (denuncia de actos delictivos por parte de los empleados), la comunicación, el almacenamiento y procesamiento de datos masivos, y la participación comprometida de las personas interesadas, ofrece a la empresa la posibilidad de escrutar su dimensión moral, emocional y responsable para estar a la altura de aquello que el momento histórico exige.

\section{Palabras clave}

Ética empresarial; Ética de las cosas; Empresa monitorizada; Bienes intangibles; Responsabilidad social; Datos masivos; Big data; Open big data; Whistleblowing.

\section{Abstract}

The big data revolution is a challenge and opportunity for companies. In the ethics and social responsibility domain, big data, and the increasingly more accessible and versatile data processing technology, allow companies to open up new communication channels, and to collect accurate, detailed, and constantly updated information about: their economic, social, 
and environmental impacts; interests and legitimate expectations involved; trust, reputation, and affinity that they arouse in their stakeholders; and the effects that their actions and decisions cause, among many other things. The objective of this study is to present a system that monitors and fulfills ethics and social responsibility which, based on whistleblowing, communication, storage, and processing of big data, along with stakeholders' committed participation, offers companies the chance to examine their moral and emotional responsibilities in order to be able to rise to what this historic time demands.

\section{Keyworks}

Business ethics; Ethics of things; Intangible assets; Monitored business; Social responsibility; Big data; Open big data; Whistleblowing.

Calvo, Patrici; Osal, Cristian (2018). "Whistleblowing y datos masivos: monitorización y cumplimiento de la ética y la responsabilidad social". El profesional de la información, v. 27, n. 1, pp. 173-184.

https://doi.org/10.3145/epi.2018.ene.16

\section{Introducción}

El informe Edelman Trust barometer, que recopila datos de más de 34.000 encuestas en 28 países, ha mostrado en los últimos años (Edelman, 2015; 2016; 2017) varias cuestiones importantes:

- Relación intrínseca entre confianza y beneficio empresarial. En sentido negativo, el $68 \%$ de los encuestados se niega a comprar productos o servicios de empresas que no les merecen confianza, y las desaconseja a conocidos, amigos o familiares. En sentido positivo, el $80 \%$ suele comprar a aquellas que les merecen confianza, y las recomiendan a sus conocidos.

- Rol social de la empresa. La gran mayoría de encuestados $(80 \%)$ creen que la organización debe jugar un papel importante en la resolución de problemas sociales, con acciones que al mismo tiempo incrementen sus beneficios. Es un dato relevante, puesto que no piden acciones altruistas. Entienden la lógica de las empresas y piden que éstas busquen el beneficio empresarial con acciones que además de incrementar sus beneficios tengan repercusiones positivas en su entorno social y medioambiental (Edelman, 2016).

- Papel de la comunicación en el ámbito empresarial. Es un recurso que permite a la organización gestionar bienes intangibles, que son importantes para su supervivencia y desarrollo, como la confianza.

\section{La responsabilidad social se ha converti- do en una pieza fundamental de la ges- tión empresarial}

Los resultados de los estudios varían significativamente cuando se separan las respuestas del "público informado" (el que tiene estudios o se preocupa por la situación económica) y del "público masa" (el que se despreocupa de la situación económica actual y de lo que se está haciendo o se podría hacer para mejorarla). La confianza del público informado en instituciones, organizaciones, líderes, medios y empresas es hasta 15 puntos porcentuales superior a la del público masa (Edelman, 2016; 2017).
Estos datos sugieren que:

- las empresas deben reconocer que su supervivencia y desarrollo depende de una correcta gestión de los bienes intangibles;

- esta gestión requiere un dialogo fluido con los stakeholders para conocer cuáles son los intereses en juego, informar de las respuestas a tales expectativas, y averiguar el grado de satisfacción de sus acciones (Edelman, 2015; 2016; 2017).

Este artículo ofrece orientaciones para crear un sistema de monitorización de la ética y la responsabilidad social que permita a una empresa escrutar su dimensión moral, emocional y de responsabilidad. Esto guarda relación con la potenciación de bienes intangibles como la confianza, la reputación o la reciprocidad, que permiten el correcto desarrollo de su actividad y la satisfacción de un máximo beneficio empresarial. Para ello:

1) Se muestra la responsabilidad social éticamente fundamentada como una forma inteligente y eficaz de gestionar la empresa. Especialmente porque aporta orientaciones para la acción y recursos comunicativos para la gestión óptima de los bienes intangibles.

2) Se proponen mecanismos de comunicación de la ética y la responsabilidad social cuya complementación permiten una gestión sostenible de la empresa.

3) Se muestra la posibilidad de monitorizar la ética y la responsabilidad social de la empresa a través de las nuevas tecnologías de almacenamiento, procesamiento y análisis de datos masivos.

4) Se ofrecen orientaciones para el diseño de un sistema de monitorización y cumplimiento de la ética y la responsabilidad social. Este sistema está vinculado a la idea original de whistleblowing (monitorizar y alertar), se basa en datos masivos abiertos, y permite gestionar y potenciar la confianza y otros bienes intangibles.

\section{Empresa plural: participación, rendición de cuentas y corresponsabilidad}

La responsabilidad social se ha convertido en una pieza fundamental de la gestión empresarial. Entre otras cosas, porque permite a las empresas: 
- mejorar el conocimiento de sí mismas;

- adaptarse mejor y más rápidamente a los cambios;

- gestionar los bienes intangibles implicados en el correcto desarrollo y potenciación de su actividad;

- minimizar los costes de transacción;

- promover un clima laboral adecuado;

- realizar un control efectivo de la conflictividad subyacente;

- orientar de manera justa y responsable las acciones y decisiones...

Aunque existen antecedentes vinculados con la filantropía y el mecenazgo, la bibliografía especializada toma como punto de partida de la responsabilidad social la década de los cincuenta del siglo pasado. Concretamente, cuando la sociedad norteamericana empezó a exigir a la empresa que diera razón del uso de su poder de influencia en los ámbitos económico, social, político o cultural (González-Esteban, 2001). Como explica Howard R. Bowen en Social responsibilities of the businessman, es una forma de entender la empresa vinculada con:

"las obligaciones de los empresarios de seguir las políticas, para tomar esas decisiones, o para seguir las líneas de acción que son deseables en términos de los objetivos y valores de nuestra sociedad" (Bowen, 1953, p. 6).

A través del concepto de responsabilidad social, Bowen llama la atención sobre dos modelos de empresa de su época que no se ajustaban bien a los nuevos tiempos:

- enfoque neoclásico (por anacrónico): basado en el marginalismo económico: sólo tenía en cuenta los intereses individuales del empresario o accionista;

- enfoque democrático (por insuficiente): basado en la democracia industrial: iba más allá del interés individual del propietario para incluir las expectativas colectivas de los empleados.

Para Bowen la responsabilidad social empresarial no tiene que ver ni con lo que los empresarios desean o consideran como bueno, ni con un equilibrio entre dos grupos de intereses internos supuestamente dicotómicos. Debe ser la sociedad la que determine qué es lo preferible, no el empresario. Cuando la empresa realice acciones para generar beneficio, debe tener en cuenta por encima de todo el interés general de la sociedad, tanto los intereses en juego como las normas aplicables (Bowen, 1953, p. 120).

Davis desarrolló las principales ideas de Bowen durante la década de los sesenta y setenta del siglo XX. Dejó de lado el sentido filantrópico de la responsabilidad social para centrarse en la relación existente entre poder y responsabilidad en los negocios.

En un primer momento, Davis (como Bowen) centró su atención en la responsabilidad del propietario, no de la empresa, y propuso en "Can business afford to ignore social responsibilities?" la idea de que el poder de éste y su responsabilidad van de la mano y exigen proporcionalidad en ambos sentidos (Davis, 1960, p. 71). Posteriormente en "Business and its environment", junto a Blomstrom, realizó una primera aproximación a lo que denominó Ley de hierro de la responsabilidad:

"Si el poder y la responsabilidad tienden hacia un es- tado de equilibrio a largo plazo, entonces el evitar la responsabilidad social conduce a la erosión gradual del poder social. Esta es la Ley de hierro de la responsabilidad: aquellos que no toman el poder en última instancia tienden a perderlo" (Davis; Blomstrom, 1966, p. 174).

Finalmente, Davis desarrolló esta Ley en "The case for and against business assumption of social responsibilities" (1973) centrando su atención en la empresa y no en el empresario e interesándose sobre todo en la reconstrucción de las condiciones de posibilidad del poder:

"La sociedad concede legitimidad y poder a la empresa. En el largo plazo, aquellas que no usan ese poder de un modo que la sociedad considera responsable tienden a perderlo" (Davis, 1973, p. 314).

A través de las ideas de Bowen, Davis y otros pensadores de mediados del siglo XX, la responsabilidad social se emancipó de las acciones filantrópicas y de mecenazgo de los empresarios, para empezar a concebirse como la justificación que hace la empresa de la gestión del poder que la sociedad le concede para llevar a cabo su actividad y obtener beneficios. El foco de atención de la responsabilidad social pasó de la preocupación sobre qué hace la empresa con parte del beneficio obtenido, al interés sobre la manera como la empresa ha logrado generar ese beneficio (Davis, 1975, pp. 19-24).

Durante los años 70 surgieron dudas sobre la posibilidad de implementación de la responsabilidad social sin la participación activa y comprometida de los empleados. A raíz de importantes escándalos relacionados con la administración pública y la empresa privada, la sociedad civil empezó a exigir la implicación de los profesionales en el escrutinio del comportamiento ético y responsable de la empresa para prevenir los efectos negativos derivados de una mala praxis por parte de propietarios y directivos (Nader; Petkas; Blackwell, 1972).

Esta inquietud generó por un lado el nacimiento del término whistleblowing para identificar las acciones de monitori-

\section{¿Preocupado por un mal proceder en tu empresa?}

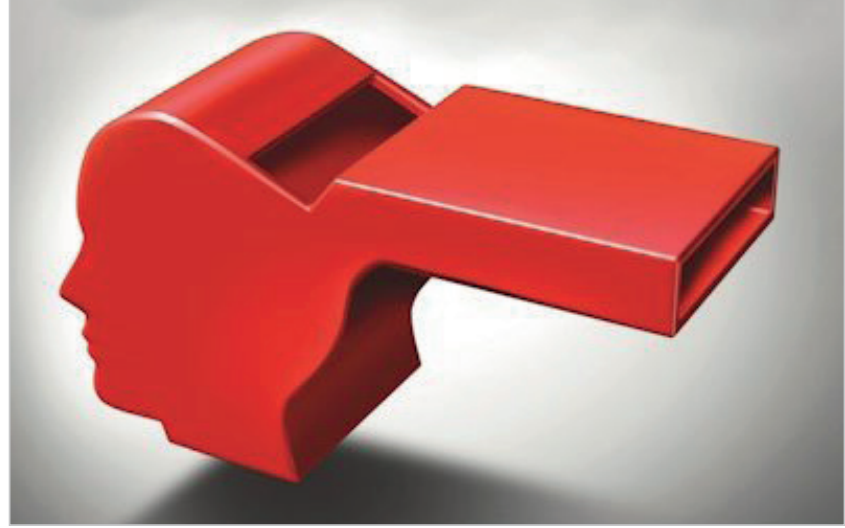

http://www.caerphilly.gov.uk 
zación y denuncia de empleados y profesionales del sector para salvaguardar la salud tanto de la organización como de la sociedad. Por otro, los primeros intentos de concreción práctica del whistleblowing a través del diseño y aplicación de sistemas de cumplimiento del comportamiento ético-legal (Vandekerckhove, 2006). A partir de ese momento se empezó a pensar en una responsabilidad social horizontal y corresponsable, cuyo acontecer en la práctica exige la participación comprometida de todos los stakeholders de la empresa.

La empresa necesitaba considerar un conjunto heterogéneo de intereses en juego, donde participaban múltiples grupos (stakeholders)

A partir de los años 80 se inició un cambio de paradigma en la gestión empresarial, con tres elementos clave:

- La teoría de los stakeholders propuesta por Freeman en 1984 mostró que la empresa necesitaba considerar un conjunto heterogéneo de intereses en juego, donde participaban múltiples grupos y en diferente grado, dependiendo de la especificidad de la organización. Formaba parte de estos grupos todo aquel "(...) que pueda afectar o ser afectado por el logro de los objetivos de la empresa" (Freeman, 1984, p. 24) y eran los responsables del poder de la empresa. Por ello la gestión de ese poder exige a la empresa justificar ante sus stakeholders o partes implicadas cómo lo están utilizando.

- El triple balance de resultados (triple bottom line) que Elkington propuso en "Towards the sustainable corporation: Win-win-win strategies for sustainable development" (Elkington, 1994) y desarrolló en Cannibals with forks: The triple bottom line of $21^{\text {st }}$ century business (Elkington, 1997). Elkington, apoyándose en el informe "Our common future: Brundtland report" (United Nations, 1987), instó a que las empresas basaran su estrategia en la sostenibilidad; es decir, que buscaran el beneficio económico al mismo tiempo que el desarrollo social y la calidad medioambiental.

- El desarrollo de la teoría de los stakeholders durante los años noventa del siglo XX. Como propuso Freeman, esta teoría señala que entre el conjunto de intereses en juego de la empresa, coexisten expectativas estratégicas de los stakeholders de carácter particular y colectivo cuya satisfacción permite mejorar la gestión estratégica de la organización. Lo que concreta y define el contenido de la responsabilidad social (y permite legitimar cómo usa su poder) es el diálogo y el posible acuerdo de todos los afectados por las acciones y decisiones de la empresa; es decir, la satisfacción de las expectativas legítimas y comunes a todos ellos (González-Esteban, 1999; 2001; 2002; 2007; 2012).

Estas propuestas teóricas abrieron la puerta a un nuevo paradigma empresarial: la empresa plural, cuya legitimidad remite al diálogo y al acuerdo intersubjetivo de todos los implicados internos y externos sobre la satisfacción de las expectativas en materia económica, social y medioambiental.
Tanto la responsabilidad social como el nuevo modelo de empresa plural empezaron a consolidarse durante la primera década del siglo XXI. En 2000 la Unión Europea decidió

“(...) convertirse en la economía basada en el conocimiento más competitiva y dinámica del mundo, capaz de crecer económicamente de manera sostenible con más y mejores empleos y con mayor cohesión social" (Lisbon European Council, 2000).

En 2001 la Comisión Europea resolvió en “Green paper: Promoting a European framework for corporate social responsibility" (Commission of the European Communities, 2001) que la forma más inteligente de alcanzar el objetivo estratégico establecido en la Cumbre Europea de Lisboa de 2000 era promoviendo la responsabilidad social entre su tejido empresarial. Es decir, promoviendo la asunción voluntaria por parte de las empresas tanto de las preocupaciones sociales y medioambientales como de procesos de diálogo con sus stakeholders internos y externos. Mediante el Libro verde se propuso una responsabilidad social para el siglo XXI basada en cuatro cuestiones básicas: voluntariedad, triple rendición de cuentas, diálogo con los stakeholders y corresponsabilidad.

Actualmente la responsabilidad social ha ganado en importancia tanto para la empresa como para la sociedad. Principalmente, porque se ha convertido en un elemento clave para la satisfacción de los 17 Objetivos de desarrollo sostenible (ODS), adoptados en la resolución de la Asamblea General de la ONU el 25 de septiembre de 2015. Éstos instan a las empresas a realizar esfuerzos conjuntos para minimizar las desigualdades, generar un crecimiento económico sostenible, erradicar el hambre y la pobreza, y producir y consumir de forma responsable, entre otras muchas cosas (United Nations, 2015). Aquí la responsabilidad social juega un papel relevante, sobre todo porque aporta a la empresa herramientas, pautas y orientaciones para hacerlo de forma eficaz y potenciando al mismo tiempo bienes intangibles como la confianza, la reputación, la afinidad o la reciprocidad.

\section{La responsabilidad social y el nuevo mo- delo de empresa plural empezaron a consolidarse durante la primera década del siglo XXI}

Para ello es necesario que la empresa implemente una responsabilidad social acorde a los tiempos actuales; es decir, que no se preocupe únicamente por mejorar la gestión estratégica de la empresa, sino también de promover una cultura empresarial ética, sostenible y responsable.

Una responsabilidad social para el siglo XXI es aquella que en el marco de una ética ciudadana evite su uso meramente estratégico y fomente procesos de diálogo con sus stakeholders que justifiquen y legitimen sus acciones y decisiones. Pero también que promueva espacios de relación para gestionar las emociones prosociales y los sentimientos y valores morales implicados. Por ello hoy se trabaja en el diseño e implementación de mecanismos de comunicación que lo posibiliten, como códigos, comités, auditorías, informes y líneas. 


\section{Mecanismos de comunicación para la gestión de la ética y la responsabilidad social empresarial}

La responsabilidad social en el marco de una ética ciudadana ofrece a la empresa mecanismos de comunicación autónomos y complementarios para el diálogo con los stakeholders internos y externos. A través de éstos, la organización busca el acuerdo intersubjetivo que posibilita su correcto desarrollo, subsistencia y máximo beneficio empresarial, la generación y potenciación de las emociones prosociales que permiten su acontecer práctico, y la consolidación de una cultura empresarial acorde con los tiempos actuales. Para entre otras cosas generar y potenciar la confianza, la reputación y los afectos de las personas que se relacionan con ella.

Entre los distintos mecanismos de comunicación destacan:

- códigos de ética y conducta;

- comités de ética y responsabilidad social;

- informes de ética y responsabilidad social;

- auditorías de ética y responsabilidad social;

- líneas de ética y responsabilidad social;

Todos ellos han sido desarrollados y sistematizados por García-Marzá en los últimos 25 años (García-Marzá, 2017; 2004; 1994).

Los códigos de ética y conducta son documentos formales donde la organización hace público su compromiso con un desarrollo ético y sostenible de su actividad. Este tipo de códigos fueron elaborados por primera vez en el siglo XVI para orientar la praxis médica ${ }^{1}$. Sin embargo, actualmente se han convertido en una herramienta fundamental en cualquier ámbito de actividad, también el económico. A través de ellos la empresa comunica a sus grupos de interés cuál es su carácter, su predisposición a hacer las cosas de una forma y manera concreta conforme a unos valores morales específicos, así como las normas que regulan su actividad y los compromisos que están dispuestos a asumir para su implementación (García-Marzá, 2017; 2004; Lozano-Aguilar, 2007; 2004). Con ello la empresa pretende abordar cuestiones tan importantes para su desarrollo como la cohesión interna, la coherencia comportamental, la construcción de la identidad, la concreción de la cultura empresarial, la diferenciación de mercado, etcétera.

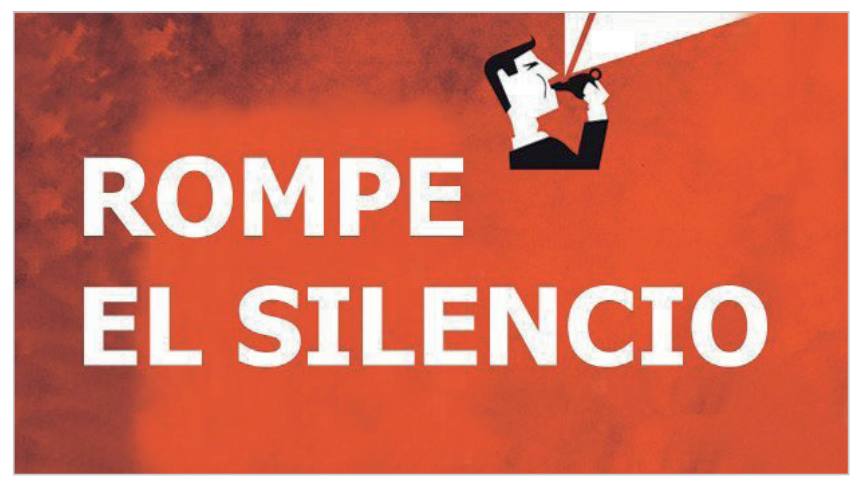

https://www.jobstreet.com.my
Los comités de ética y responsabilidad social son el principal espacio de diálogo y deliberación de los stakeholders de la organización. Estos comités emergieron en el entorno sanitario durante la segunda mitad del siglo XX, cuando en 1953 los National Institutes of Health exigieron que toda investigación con sujetos humanos que fuese realizada en sus instalaciones contara con la aprobación previa de un comité de expertos que velara por su protección: los llamados Institutional review boards ${ }^{2}$. Actualmente los comités de ética se han extendido a diferentes áreas de actividad, como la económica (Cortina, 1996). Actúan como un medio de comunicación cuya principal función es asesorar, realizar informes, gestionar las notificaciones de alertas, denuncias, sugerencias y propuestas de mejora, colaborar en la resolución de conflictos, concienciar sobre la necesidad de ser económica, social y medioambientalmente sostenibles, formar a los empleados en el código ético, promover la cultura empresarial y supervisar los informes de responsabilidad social, entre otras cosas.

Las memorias de ética y responsabilidad social son documentos formales donde la empresa hace públicas sus normas sobre sostenibilidad económica, social y medioambiental

Las memorias de ética y responsabilidad social son documentos formales donde la empresa hace públicas sus normas sobre sostenibilidad económica, social y medioambiental. Nacidas durante la década de los 80 y 90 del siglo XX y promovidas conjuntamente por Pnuma (Programa de Naciones Unidas para el Medio Ambiente) y Ceres (Coalition for Environmentally Responsible Economies) a partir de 1997, las memorias de responsabilidad social constituyen un medio de comunicación entre la empresa y sus stakeholders. Su principal función es generar transparencia, reputación, confianza, afinidad, autoconocimiento, mejoramiento, ajuste estructural, visualización de potencialidades y debilidades, control y prevención de conflictos, mejora de la satisfacción laboral, adaptación rápida a los nuevos retos, buena imagen de marca, minimización de costes de transacción... Actualmente existen varias metodologías para la elaboración de memorias, entre las cuales destaca por su mayor utilización, actualización y claridad Global reporting initiative, que presenta indicadores para la medición, divulgación y rendición de cuentas de la empresa en materia de responsabilidad social.

Las auditorías de ética y responsabilidad social son procesos de valoración y comunicación del grado de cumplimiento de los compromisos alcanzados por la empresa, certificando la coherencia existente entre lo que se dice y lo que se hace, o como argumenta García-Marzá:

“(...) mostrar que la organización 'va en serio', que la responsabilidad social no es una mera estrategia, sino que forma parte de su ética, esto es, de su carácter o forma de ser y hacer" (García-Marzá, 2017, p. 274). 
Para su implementación ${ }^{3}$, García-Marzá propone una metodología basada en la teoría de la acción comunicativa y la ética discursiva de Jürgen Habermas, donde las perspectivas del observador y el participante se combinan para aportar datos objetivos y percepciones sobre la realidad empresarial (García-Marzá, 2017, p. 274). Desde esta doble perspectiva, Rodríguez et al. (2006) desarrollaron un modelo de auditoría de ética y responsabilidad social basado en variables generales, indicadores concretos y medibles, y distintas fases y etapas de la auditoría (preparación, trabajo de campo e informe final).

Las líneas de ética y responsabilidad social son canales de comunicación establecidos por la empresa para que los stakeholders puedan opinar, alertar, denunciar y aportar soluciones y/o sugerencias sobre el nivel moral y responsable de ésta (García-Marzá, 2017). Están basadas en los sistemas whistleblowing que emergen a partir de 1971 y se consolidan en 2002 con la Ley Sarbanes-Oxley (SOX) aprobada por el Congreso Norteamericano como medida de prevención y control de las sociedades cotizadas (Congress of the United States of America, 2002).

Su principal función es sistematizar las notificaciones sobre el incumplimiento de los compromisos y exigencias de la organización en materia de responsabilidad social, así como recabar información sobre las propuestas de los stakeholders relacionadas con la vigencia del código ético y de conducta, la revisión de las políticas de responsabilidad social, la adecuación de los mecanismos que permiten su implementación y correcto desarrollo, la veracidad y calidad de la memoria de responsabilidad social, o la mejora y correcto funcionamiento de la propia línea de responsabilidad social, entre otras cosas (García-Marzá, 2017; Calvo, 2015a; 2015b; 2016a; 2016b). El comité de ética y responsabilidad social, en tanto que espacio de diálogo y deliberación de los stakeholders de la empresa, actúa como receptor y gestor de la línea ética.

Estos mecanismos y herramientas de gestión de la comunicación debidamente complementados (García-Marzá, 2017), permiten a la empresa hacerse cargo de su responsabilidad y gestionar la dimensión moral que la subyace. De ello depende la generación y desarrollo de los bienes intangibles implicados en el logro de un máximo beneficio empresarial.

La actual revolución de los datos masivos (big data) es un reto para las organizaciones al menos por tres cuestiones:

- la brecha informacional entre quienes están a favor del uso de datos masivos y quienes no, como elemento distorsionador en la economía durante los próximos años;

- la gestión de intangibles como confianza, reputación, reciprocidad o afinidad depende de la gestión de la dimensión tanto estratégica como moral y emocional de la empresa, y una monitorización nula o inadecuada se convierte en un factor disruptivo;

- la monitorización está al alcance tanto de la empresa como de las personas relacionadas con la misma. Ésta puede utilizar los programas de análisis de datos masivos para conocer mejor los impactos que produce su actividad y las necesidades, expectativas, intereses y afectos de sus

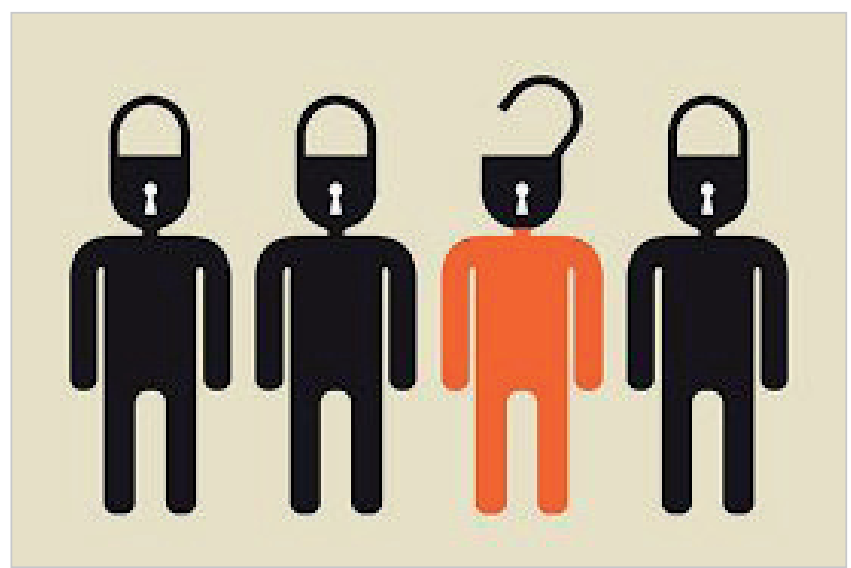

https://www.ibanet.org

grupos de interés. Y éstos, gracias a una tecnología cada vez más accesible y gratuita, y a la emergencia de webs de asesoramiento (business advisors), están empezando a escrutar el ámbito empresarial para tomar decisiones mucho más beneficiosas, justas y prudentes.

\section{Empresa plural en la era de los datos masivos}

Durante la Conference on professional responsibility celebrada en Washington D.C. el 30 de enero de 1971, el activista político Ralph Nader realizó una llamada a la responsabilidad de los profesionales para que monitorizasen a sus empresas y alertasen cuando tuvieran constancia de prácticas destructivas o inmorales que pudieran dañar la salud de éstas o de la sociedad. Este toque de atención fue el punto de partida del término whistleblowing (dar la voz de alerta). El neologismo acuñado por el propio Nader define las actitudes comprometidas de directivos y empleados, que anteponen el interés general de la sociedad al interés particular de la organización pública o privada en la cual desarrollan su actividad (Boffey, 1971, pp. 549-551), pero también para evitar que este tipo de acciones fueran vinculadas con términos peyorativos como chivato o soplón (Nader; Petkas; Blackwell, 1971).

Tras casi medio siglo de teorización conceptual y práctica ${ }^{4}$, whistleblowing ha logrado despertar el interés de las empresas gracias al apoyo de normas, códigos y directrices nacionales e internacionales, especialmente las vinculadas con la responsabilidad social ${ }^{5}$. Entre otras, la norma ISO 26000 sobre responsabilidad social, que en su apartado 6.3.6 Asunto 4 sobre derechos humanos: resolución de reclamaciones y 4.4 Compromiso ético promueve:

"el establecimiento y el mantenimiento de mecanismos para facilitar que se informe sobre comportamientos no éticos sin miedo a represalias" (ISO, 2010).

También la Global reporting initiative G.4 (GRI, 2014), que a través de los indicadores G.4-49, G.4-57 y G.4-58 insta a las empresas a que reporten sobre los sistemas de cumplimiento que utilizan para el desempeño de los objetivos en responsabilidad social. Y sobre todo los GRI Sustainability reporting standards (GRI, 2016) que deben ser aplicados en los informes publicados a partir de julio de 2018, en cuyos 
estándares 205 Anticorrupción, 307 Cumplimiento ambiental, y 419 Cumplimiento socioeconómico se establecen los requerimientos de notificaciones sobre prácticas corruptas (soborno, pagos por facilitación, fraude, extorsión, colusión, blanqueo de capitales o malversación), y de incumplimiento de leyes o normativas concretas sociales, económicas y medioambientales (como los 17 Objetivos de desarrollo sostenible).

Sin embargo estas normas, guías y estándares han derivado hacia una implementación sesgada y deficitaria del whistleblowing en las empresas. Instan a realizar esfuerzos en la implantación de canales de alertas y denuncias de irregularidades ético-legales, pero no dicen nada de la necesidad de implementar sistemas de monitorización que permitan una vigilancia proactiva y constante del comportamiento esperado mediante la apertura de canales de comunicación entre la empresa y sus stakeholders. Con ello desprecian su potencial como elemento transformador de la realidad económica y social, así como su capacidad para gestionar los recursos intangibles necesarios.

En responsabilidad social existen diversas aplicaciones de análisis de datos masivos que la empresa puede usar para monitorizar aspectos globales o concretos

En un sentido muy acotado, por monitorizar se entiende:

“Observar mediante aparatos especiales el curso de uno o varios parámetros fisiológicos o de otra naturaleza para detectar posibles anomalías" (Real Academia Española, 2014).

En un sentido amplio, como proponen Nader y otros teóricos del whistleblowing, la monitorización abarca aspectos legales, sociales, medioambientales y morales. Por ejemplo el escrutinio de:

- grado de satisfacción de las expectativas legítimas en juego;

- impactos económicos, sociales y medioambientales de su actividad;

- afectos de los grupos de interés;

- nivel de confianza, afinidad o reputación alcanzado;

- la prevención de prácticas ilegales...

Es decir, monitorizar como acción de proteger, respetar y remediar, como orientan los principios rectores del Marco Ruggie $^{6}$, así como de llevar a cabo una actividad empresarial en el marco de los Objetivos de desarrollo sostenible (ODS) (United Nations, 2011; 2015).

En el pasado la falta de interés de las normas, guías y estándares por la monitorización pudo deberse a la complejidad y falta de recursos tecnológicos para abordar con cierta garantía la monitorización de la responsabilidad de la gestión empresarial. Cuando en 1971 Nader apuntaba en esta dirección, internet era todavía un proyecto de estado en Norteamérica. Hoy la posibilidad de almacenar grandes volúmenes de datos, la disponibilidad de softwares de análisis de datos masivos y el rápido avance de internet de las cosas (internet of things) proporciona información mucho más precisa sobre las tendencias, exigencias, intereses, expectativas, comportamientos, motivaciones y emociones de los grupos de interés internos y externos (Yager; Pascual-Espada, 2017; Kocovic et al., 2017; Tascón; Coullaut, 2016).

La monitorización no sólo es posible, sino necesaria y exigible para poder llevar a cabo una actividad económica de forma eficiente, eficaz y responsable.

Las tecnologías de datos masivos hacen real, actual e irrenunciable la capacidad de articular cantidades extraordinarias de datos desde una numerosa cantidad de fuentes, así como la capacidad de generar información relevante a partir de tales datos. En responsabilidad social existen diversas aplicaciones de análisis de datos masivos que la empresa puede usar para monitorizar aspectos globales o concretos. Algunas son de fácil acceso y a muy bajo coste, por ejemplo:

- para el análisis de documentación, legislación e informes relacionados con la responsabilidad social, destaca $B i$ gQuery de Google, una plataforma online de almacenamiento de datos que integra un software de análisis datos masivos;

- para el análisis del nivel de compromiso de los grupos interesados, Kaushik, que utiliza una métrica basada en la identificación de las motivaciones, emociones y sentimientos que hay detrás de la confianza, la reputación, la afinidad o la cohesión;

- para el análisis de la reputación corporativa, destacan Asomo, que combina técnicas de análisis semántico y de crowdsourcing; Socialmention, que calcula la reputación de la empresa en las redes sociales; y BlogPulse, que monitoriza contenidos de blogs para conocer la imagen de la empresa;

- para el análisis interno y externo de las emociones y los sentimientos relacionados con la empresa y su actividad destacan Klout y Peerlndex, que rastrean datos de millones de espacios externos y en la información de la propia empresa; y Ciao, Swotti y TwitsObserver, que realizan minería de opinión para extraer información relevante de los comentarios realizados por los stakeholders en foros, webs, blogs, redes sociales, etc.;

- para investigación, seguimiento y control (monitorización) de las actitudes de la organización y de los stakeholders destacan Socialmention, Google Insights, Social Report, Twitter Search, y Sysomos, entre otras muchas ${ }^{7}$.

Estas aplicaciones permiten una gestión adecuada de la responsabilidad de la empresa y de las motivaciones que le subyacen. Con su uso sistematizado, la organización conoce en tiempo real los intereses en juego, así como los impactos y conflictos en materia económica, social y medioambiental.

Asimismo favorecen una nueva concepción de memorias de responsabilidad social, una nueva forma de rendir cuentas. Las herramientas de análisis de datos masivos fomentan las reporting apps o triple bottom apps. Estas aplicaciones ofrecen información en tiempo real de los impactos económicos, sociales y medioambientales de la empresa mediante el procesamiento de datos internos y externos y su conversión en gráficos de burbujas, cartogramas, mapas, árboles de nodos, diagramas de Sankey, ruedas de acordes, matrices, 
etc. De esta forma las memorias pasan a ser más completas y comprensibles, pero sobre todo, participativas, puesto que la organización y los grupos de interés compartirán en diferente grado el protagonismo y la responsabilidad en la elaboración de la memoria.

La empresa es la responsable de diseñar y ofrecer en abierto una plataforma 3.0 de recogida, procesamiento y comunicación de datos. Organización y grupos de interés contribuyen en los contenidos de la memoria con información relevante que suben a la plataforma (caso de la empresa) o mediante noticias, opiniones y notificaciones en periódicos, webs, foros, blogs, redes sociales, líneas de responsabilidad social... (caso de los stakeholders).

Esta nueva concepción de memorias de responsabilidad social (abierta y participativa) permite ver de forma rápida, clara y en tiempo real:

- qué esfuerzos ha realizado o está realizando la empresa para subsanar deficiencias, carencias o malas acciones y decisiones;

- cuál es el grado de satisfacción de los intereses en juego;

- qué afectos despierta entre los grupos de interés;

- qué nuevos conflictos emergen.

También permite confrontar la veracidad de la información publicada en la memoria gracias al análisis de la información de fuentes externas, como por ejemplo las business advisors, webs que recopilan y procesan información directamente proporcionada por los propios empleados de las empresas.

Entre otras muchas, cabe destacar Glassdoor, que aporta datos que van más allá de cómo valoran los stakeholders la empresa, adentrándose en la confianza, la afinidad, la reciprocidad, la justicia o la responsabilidad interna. Entre otras cuestiones importantes, Glassdoor proporciona información interesante sobre oportunidades, salarios, conciliación, cultura, calidad y valores de la empresa, así como en qué grado los empleados recomendarían a un amigo trabajar en ella.

Otras business advisors similares, aunque menos desarrolladas en la actualidad, están empezando a recabar y procesar datos y opiniones de clientes, proveedores, organizaciones de la sociedad civil y demás grupos interesados, ofreciendo información valiosa sobre satisfacción en los aspectos económicos, sociales y medioambientales de la empresa.

Todas estas cuestiones han despertado el interés efectivo de las grandes compañías por las técnicas de datos masivos, así como el interés latente de pequeñas y medianas empresas y de los stakeholders que esperan la emergencia de una nueva tecnología inclusiva y accesible que les permita participar de sus expectativas y beneficios.

Sin embargo, el uso actual de datos masivos en el ámbito empresarial dista mucho de ser el adecuado.

La monitorización de las empresas a través de los datos masivos que producen directa o indirectamente, queda lejos del sentido propuesto por Nader y otros teóricos del whistleblowing, principalmente porque su uso es unidireccional, excluyente e instrumental. Es decir, las empresas con suficiente poder para hacerse cargo de ello están por un lado aplicando mecanismos de control de datos masivos (information governance) que limitan, tergiversan o distorsionan la información disponible en su propio beneficio, y por otro, utilizando el análisis de los datos masivos como un modelo predictivo del comportamiento que les permita optimizar el beneficio mediante el control de la voluntad libre o el mero aprovechamiento de las matrices de opinión de los grupos de interés, especialmente de los clientes.

Ambas cuestiones sesgan y pervierten el sentido de la monitorización (Feenstra, 2012; Feenstra et al., 2017), generando recelo entre competidores, promoviendo una mayor opacidad en el mundo empresarial, y fomentando la desconfianza de los stakeholders. Se ve socavado su potencial como instrumento no sólo adaptativo, sino transformador de la realidad social (Feenstra, 2015a; 2015b), así como su capacidad para gestionar la confianza, la reputación, la afinidad o la reciprocidad (Calvo, 2016b; 2016c).

En lo que respecta a internet de las cosas, los continuos casos de mala praxis y el relativismo conductual de los algoritmos de comportamiento está generando un nuevo campo de investigación (la ética de las cosas): abarca el estudio del comportamiento esperado de los dispositivos que recaban información y se nutren de datos masivos, y exige monitorización. Por ejemplo, estudia si es lícito que los robots domésticos estén programados para recabar información de sus dueños, como los planos de su casa o sus hábitos o pautas de comportamiento, sin el consentimiento explícito de sus propietarios. $\mathrm{O}$ si los coches sin conductor deben anteponer la vida de sus pasajeros a la de los viandantes en caso de peligro por accidente, entre otras muchas cosas.

Todo ello está produciendo la emergencia de nuevos conceptos y campos de estudio, como ética algorítmica, ética de datos, gobierno algorítmico, entre otros ${ }^{8}$, así como la exigencia de una monitorización adecuada que prevenga los posibles daños derivados.

Por estos y otros motivos, es necesario trabajar en el diseño y aplicación de sistemas de monitorización y cumplimiento que mediante la combinación de herramientas de análisis

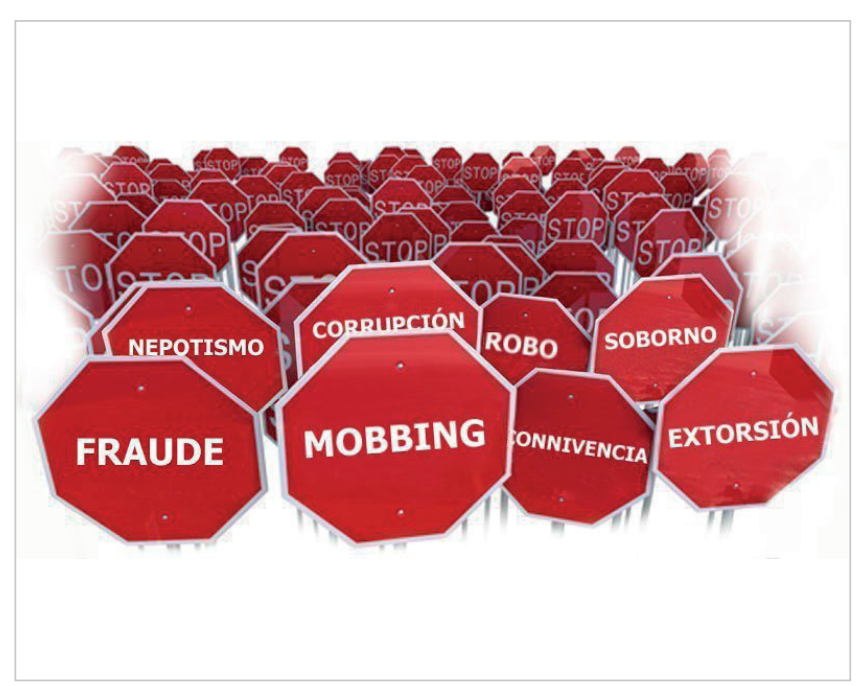

http://www.cput.ac.za/whistle-blowing 
de datos masivos con otros instrumentos como códigos éticos y de conducta, permitan a la empresa un uso adecuado de los datos masivos y la inclusión de los stakeholders en el escrutinio del comportamiento de la empresa.

\section{Sistema de monitorización de la ética y la responsabilidad social empresarial}

Utilizado de forma responsable, el análisis de los datos masivos ofrece a la empresa la posibilidad de monitorizar los impactos de su actividad, los conflictos e intereses en juego, los afectos que despierta entre sus grupos de interés, entre otras cosas. Esto proporciona a la empresa una valiosa información para:

- realizar su actividad a la altura de lo que el tiempo actual exige;

- tomar decisiones más racionales y responsables;

- gestionar los bienes intangibles necesarios para llevar a cabo su actividad de manera eficiente y sostenible.

El análisis de los datos masivos también puede fomentar que la empresa deje en manos del "capricho tecnológico" su rumbo presente o futuro, así como un uso inadecuado o irresponsable de la información disponible (Suárez-Gonzalo, 2017; Tascón; Coullaut, 2016). Para evitarlo, es necesario un sistema de monitorización y cumplimiento que sea capaz de sacar el máximo partido a los datos masivos mediante una gestión efectiva, eficiente y responsable. Siguiendo directrices y estándares internacionales, así como bibliografía especializada ${ }^{9}$, este sistema de monitorización y cumplimiento debería contener al menos seis pasos:

\section{Protocolo de gestión y actuación}

Es el documento guía que recoge toda la información relacionada con los procesos y acciones del sistema. Entre ellos:

- recursos disponibles;

- estructura del sistema;

- plan básico de gestión y actuación del sistema;

- plan para el despliegue e implementación del sistema (plan de comunicación, plan de responsabilidad social, plan de procesos...);

- normas, códigos y estándares empresariales, sectoriales e internacionales que constituyen el marco normativo del sistema (valores, principios y normas de la empresa, de la profesión y del sector; norma ISO26000 sobre responsabilidad social; Ley de responsabilidad social; Ley de protección de datos, política de responsabilidad social de la empresa; principios rectores del Marco Ruggie, objetivos de desarrollo sostenible, etc.);

- documento descriptivo sobre la estructura, instrumentos y alcance del sistema de monitorización;

- programa de formación para el fomento y concienciación sobre el sistema y sus beneficios;

- otras cuestiones relevantes sobre implementación y desarrollo de los procesos de monitorización.

\section{Mecanismos de comunicación}

Canales de comunicación utilizados por la empresa para mostrar tanto su modo de hacer las cosas como los impactos económicos, sociales y medioambientales que produce su actividad y lo compromisos que es capaz de asumir. Entre ellos:
- el código ético, donde la organización muestra a los stakeholders sus valores y los compromisos que es capaz de asumir para su implementación práctica;

- el informe o memoria de responsabilidad social, que, además de comunicar a los stakeholders los impactos económicos, sociales y medioambientales mediante indicadores medibles y comparables, se postula como plataforma de comunicación de los resultados de la monitorización;

- otros mecanismos de comunicación que ayuden a abrir canales de información y diálogo con los stakeholders.

\section{Programas de análisis de datos masivos}

Conjunto de instrumentos de análisis de datos masivos seleccionados para monitorizar aspectos concretos o generales sobre la responsabilidad social de la empresa. Por ejemplo, como se ha explicado con anterioridad, herramientas de análisis de datos relacionados con:

- legislación y los informes de responsabilidad social;

- grado de compromiso y afectos (motivaciones, emociones, sentimientos y pasiones) de los stakeholders;

- nivel de confianza, reputación, afinidad o cohesión que transmite la empresa;

- intereses y expectativas en juego;

- impactos que produce la actividad de la empresa en materia económica, social y medioambiental;

- conflictos existentes y/o latentes;

- expectativas; etc.

\section{Instrumentos de gestión y cumplimiento}

Diseño y puesta en marcha de tres mecanismos de gestión y cumplimiento de la monitorización (control, veracidad y alertas) de la responsabilidad social autónomos pero complementarios y necesarios (García-Marzá, 2017):

- el comité de responsabilidad social, como espacio de participación de los stakeholders de la empresa, se ocupa de velar por el buen funcionamiento del sistema, de gestionar las sugerencias, alertas y denuncias recibidas a través de la línea de cumplimiento; de realizar informes y propuestas de mejora del sistema de monitorización; de asesorar a la empresa en la resolución de conflictos relacionados con la responsabilidad social, etc.;

- la auditoría de responsabilidad social se encarga de revisar el sistema y todos sus elementos, así como de la calidad y veracidad de la información recopilada, analizada y publicada;

- la línea de cumplimiento se preocupa de sistematizar las alertas, denuncias, sugerencias y propuestas de revisión y mejora del sistema de monitorización de la responsabilidad social.

\section{Programa de formación}

Se trata de diseñar e implementar un programa formativo para promover el sistema de monitorización entre los stakeholders y fomentar su participación comprometida en los procesos de elaboración, escrutinio y difusión de la información. Con ello se estimula el crecimiento del público informado y la confianza, la reputación y la afinidad.

\section{Responsable de la monitorización y cumplimiento}

Designar un responsable principal del seguimiento y la gestión del sistema de monitorización y cumplimiento. 


\section{Conclusiones}

El uso de datos masivos (big data) hace que la monitorización de la ética y responsabilidad social empiece a ser percibida por la empresa y los stakeholders (grupos de interesados) como algo posible y necesario.

- Desde el punto de vista de la organización, para mantenerse a la altura de las expectativas de sus stakeholders, tomar decisiones maduras, y gestionar y potenciar los bienes intangibles que son condición de posibilidad del correcto desarrollo de su actividad.

- Desde el punto de vista de los stakeholders, como instrumento para escrutar y mantenerse bien informados sobre los impactos y las acciones de la empresa y poder tomar decisiones racionales basadas en el beneficio tanto estratégico como moral, emocional y responsable.

De ahí que ambos estén exigiendo datos abiertos y acceso no restringido a los medios de escrutinio, elaboración y análisis de datos (open big data) para disfrutar de sus beneficios y evitar o minimizar los efectos negativos de la brecha informacional, de la exclusión, o de la instrumentalización que puede generar el uso irresponsable de datos masivos.

Empresa y stakeholders exigen datos abiertos y acceso no restringido a los medios de escrutinio, elaboración y análisis de datos

\section{Notas}

1. El Código de ética médica del Royal College of Physicians de 1543 está considerado el primero de la historia (Brown; Bright; Tavris, 2007, p. 128).

2. En el entorno sanitario existen otros tipos de comités: los comités de bioética asistencial, que nacieron en 1968 cuando la Harvard Medical School (EUA) creó un comité ad hoc para aclarar los supuestos de muerte cerebral (Post; Blustein, 2015, p. 133), y los comités de bioética nacionales, que aparecieron en la década de los 70 en Francia, Italia y Portugal para realizar informes, propuestas y recomendaciones a los poderes públicos (Cortina, 1996).

3. Otros estudios relevantes sobre metodología e implementación de las auditorías éticas son Rodríguez-Córdoba et al. (2006) y Lozano-Aguilar (2007).

4. Para un estudio sobre el proceso de teorización conceptual y práctica del whistleblowing, ver Calvo (2016a; 2016b).

5. Para profundizar en la relación entre el whistleblowing y la responsabilidad social, Vandekerckhove (2006).

6. Principios rectores de derechos humanos en las empresas aprobados por el Consejo de Derechos Humanos de Naciones Unidas que dan a las empresas un modelo, como marco común de implementación integral; constituyen puntos de referencia para que los grupos de interés puedan evaluar sus propios desempeños y los de su cadena de valor. Anticipan un nuevo enfoque en las políticas de contratación de los recursos humanos y abren nuevas perspectivas en el liderazgo, la legitimidad y el alcance de la licencia para operar.
7. Para más información sobre éstas y otras herramientas de análisis de datos masivos, ver los estudios de Joyanes-Aguilar (2013), Serrano-Puche (2012), Estellés-Arolas y González-Ladrón-de-Guevara (2012), Serrano-Cobos (2014), Rodríguez-Vaamonde, Torre-Bastida y Garrote (2014), Del-Fresno-García (2011), entre otros.

8. Para saber más sobre todas estas cuestiones, Monasterio-Astobiza (2017).

9. Al respecto, en el campo de las directrices internacionales destacan las recomendaciones del Consejo de Europa a través del Dictamen 1/2006 elaborado por el Grupo de Trabajo del Artículo 29 sobre Protección de Datos (2006) y los estándares BSI Whistleblowing arrangements code of practice (2008), ECS2000: A guidance document for the implementation of the ethics compliance standard 2000 (Reitaku University, 1999) y Australian Standard AS8000 (SAIC, 2011). En el campo de la bibliografía especializada, destacan los trabajos de García-Marzá (2017), Calvo (2015a; 2015b; 2016a; 2016b), Miceli, Near y Dworkin (2013), Vandekerckhove (2006), Del-Fresno-García (2011), entre otros.

\section{Agradecimientos}

Este estudio es parte del Proyecto de investigación cientifica y desarrollo tecnológico FFI2016-76753-C2-2-P, financiado por el Ministerio de Economía y Competitividad, y UJI-A2016-04, financiado por la Universitat Jaume I.

\section{Referencias}

Boffey, Philipp M. (1971). "Nader and the scientists: A call for responsibility". Science, v. 171, n. 3971, pp. 549-551. https://doi.org/10.1126/science.171.3971.549

Bowen, Howard R. (1953). Social responsibilities of the businessman. New York: Harper and Row.

British Standars Institute (2008). PAS 1998:2008. Whistleblowing arrangements. Code of practice. ISBN: 9780580 509735 https://goo.gl/jigikh

Brown, S. Lori; Bright, Roselie A.; Tavris, Dale R. (eds.) (2007). Medical device epidemiology and surveillance. Chichester, West Sussex: Wiley. ISBN: 9780470060865

Calvo, Patrici (2015a). "Economía ética hermenéutico-crítica y su gestión en las organizaciones". Revista venezolana de gerencia, v. 20, n. 71, pp. 534-553.

http://www.redalyc.org/html/290/29042408009/

Calvo, Patrici (2015b). "Responsabilidad social ético-discursiva: el papel del whistleblowing en la emergencia y desarrollo de los bienes cordiales". Neumann business review, diciembre, v. 1, n. 2, pp. 1-22.

https://goo.gl/kQWsZ3

Calvo, Patrici (2016a). “Economía con sentido moral. Un sistema de monitorización y gestión de la ética para las empresas y organizaciones". Tópicos, n. 50, pp. 211-248. https://goo.gl/w8c9vT

Calvo, Patrici (2016b). "Whistleblowing ante la miseria moral de instituciones y organizaciones". En: Meseguer-Sánchez, Víctor; Avilés-Hernández, Manuela (dirs.); Nicolás-Guardio- 
la, Juan J.; Giner-Alegría, César-Augusto; (coords.). Empresa, derechos humanos y RSC. Una mirada holística desde las ciencias sociales y jurídicas. Cizur Menor: Aranzadi Thomson Reuters. ISBN: 9788490997871

Calvo, Patrici (2016c). "Hacía una economía cordial". Veritas. Revista de filosofía y teología, n. 35, pp. 29-56. http://repositori.uji.es/xmlui/handle/10234/163596

Commission of the European Communities (2001). Green paper. Promoting a European framework for corporate social responsibility. Bruselas: European Commission.

http://europa.eu/rapid/press-release_DOC-01-9_en.pdf

Congress of the United States of America (2002). "An act to protect investors by improving the accuracy and reliability of corporate disclosures made pursuant to the securities laws, and for other purposes". Public law, n. 107-204, 116 Stat. 745, 30/07/2002.

https://goo.gl/BzFGos

Cortina, Adela (1996). "Comités de ética”. Cuestiones morales, v. 12. Madrid: Trotta, pp. 291-306. ISBN: 97884 8164-129 5

Davis, Keith (1960). "Can business afford to ignore social responsibilities?". California management review, v. 2, n. 3, pp. $70-76$.

https://goo.gl/mQVmQV

https://doi.org/10.2307/41166246

Davis, Keith (1973). "The case for and against business assumption of social responsibilities". Academy of management journal, v. 16, n. 2, pp. 312-322.

https://doi.org/10.2307/255331

Davis, Keith (1975). "Five propositions for social responsibility". Business horizons, v. 18, n. 3, pp. 19-24.

https://doi.org/10.1016/0007-6813(75)90048-8

Davis, Keith; Blomstrom, Robert L. (1966). Business and its environment. New York: McGraw-Hill. ISBN: 9780 070155206

Del-Fresno-García, Miguel (2011). "Infosociabilidad: monitorización e investigación en la web 2.0 para la toma de decisiones". El profesional de la información, septiembre-octubre, v. 20, n. 5, pp. 548-554.

https://doi.org/10.3145/epi.2011.sep.09

Edelman (2015). 2015 Edelman Trust barometer. Global annual study, Edelman Berland.

https://goo.gl/iMg9eJ

Edelman (2016). 2016 Edelman Trust barometer. Global annual study. Edelman Berland.

https://goo.gl/ReZUf2

Edelman (2017). 2017 Edelman Trust barometer. Global annual study. Edelman Berland.

https://www.edelman.com/trust2017

Elkington, John (1994). "Towards the sustainable corporation: Win-win-win strategies for sustainable development". California management review, v. 36, n. 2, pp. 90-100.

https://goo.gl/vE7iHe

https://doi.org/10.2307/41165746

Elkington, John (1997). Cannibals with forks: The triple bottom line of $21^{\text {st }}$ century business. Londres: John Wiley and

\section{Sons. ISBN: 9781841120843}

Estellés-Arolas, Enrique; González-Ladrón-de-Guevara, Fernando (2012). "Clasificación de iniciativas de crowdsourcing basadas en tareas". El profesional de la información, v. 21, n. 3, pp. 283-291.

https://doi.org/10.3145/epi.2012.may.09

Feenstra, Ramón A. (2012). Democracia monitorizada en la era de la nueva galaxia mediática. La propuesta de John Keane. Barcelona: Icaria. ISBN: 9788498884357

Feenstra, Ramón A. (2015a). “Activist and citizen political repertoire in Spain: A reflection based on civil society theory and different logics of political participation". Journal of civil society, v. 11, n. 3, pp. 242-258.

https://doi.org/10.1080/17448689.2015.1060662

Feenstra, Ramón A. (2015b). "El potencial transformador de la democracia monitorizada a debate: contextualización teórica y diálogo con John Keane". Teknokultura, v. 13, n. 2, pp. 639-654.

http://revistas.ucm.es/index.php/TEKN/article/view/52437

Feenstra, Ramón A.; Tormey, Simon; Casero-Ripollés, Andreu; Keane, John (2017). Refiguring democracy: The Spanish political laboratory. New York: Routledge. ISBN: 9781 138063686

Freeman, R. Edward (1984). Strategic management: A stakeholders approach. Pitman: Boston.

García-Marzá, Domingo (1994). “Asesoría ética en la empresa: hacia un nuevo concepto de empresa". En: Cortina, Adela (coord.). Ética de la empresa. Claves para una nueva cultura empresaria. Madrid: Trotta. ISBN: 9788481640137

García-Marzá, Domingo (2004). Ética empresarial: del diálogo a la confianza. Madrid: Trotta. ISBN: 9788481646948

García-Marzá, Domingo (2017). “From ethical codes to ethical auditing: An ethical infraestructure for social responsibility communication". El profesional de la información, v. 26, n. 2, pp. 268-276.

https://doi.org/10.3145/epi.2017.mar.13

González-Esteban, Elsa (1999). "La empresa ante sus grupos de intereses: una aproximación desde la literatura del análisis de los stakeholders". Papeles de ética, economía y dirección, n. 4, pp. 1-14.

https://goo.gl/u5bHPU

González-Esteban, Elsa (2001). La responsabilidad moral de la empresa. Una revisión de la teoría de stakeholder desde la ética discursiva. Tesis doctoral. Universitat Jaume I. https://goo.gl/AggLhS

González-Esteban, Elsa (2002). “Defining a post-conventional corporate moral responsibility". Journal of business ethics, v. 39, n. 1-2, pp. 101-108.

https://goo.gl/Kbnu29

https://doi.org/10.1023/A:1016388102599

González-Esteban, Elsa (2007). "La teoría de los stakeholders. Un puente para el desarrollo práctico de la ética empresarial y de la responsabilidad social corporativa". Veritas. Revista de filosofía y teología, v. 2, n. 17, pp. 205-224. http://www.redalyc.org/pdf/2911/291122924002.pdf 
González-Esteban, Elsa (2012). "Perspectiva de los grupos de interés en la RSE". En: Raufflet, Enmanuel; Lozano, José-Félix; Barrera, Ernesto; García-de-la-Torre, Consuelo. Responsabilidad social empresarial. Pearson: México, pp. 91-103. ISBN: 9786073209403

Global Reporting Initiative (2014). G4 Sustainability reporting guidelines. Reporting principles and standard disclosures. https://goo.gl/E4aV5F

Global Reporting Initiative (2016). Consolidated set of GRI sustainability reporting standards 2016.

https://www.globalreporting.org/standards

Grupo de Trabajo del Artículo 29 sobre Protección de Datos (2006). Dictamen 1/2006 relativo a la aplicación de las normas sobre protección de datos de la UE a los sistemas internos de denuncia de irregularidades en los ámbitos de la contabilidad, controles de auditoría internos, cuestiones de auditoría, lucha contra la corrupción y delitos financieros y bancarios. Grupo sobre Protección de Datos del Artículo 29. https://goo.gl/44DSuf

ISO (2010). Norma ISO 26000: 2010. Guía de responsabilidad social. ISO: Genova.

https://www.iso.org/obp/ui\#iso:std:iso:26000:ed-1:v1:es

Joyanes-Aguilar, Luis (2013). Big data. Análisis de grandes volúmenes de datos en organizaciones. México: Alfaomega. ISBN: 9788426720818

Kocovic, Petar; Behringer, Reinhold; Ramachandran, Muthu; Mihajlovic, Radomir (eds.) (2017). Emerging trends and applications of the internet of things. Hershey, PA: IGI Gobal. ISBN: 9781522524373

https://doi.org/10.4018/978-1-5225-2437-3

Lisbon European Council (2000). Lisbon European Council 23 and 24 March 2000. Presidency conclusions.

https://goo.gl/VzWS1W

Lozano-Aguilar, José-Félix (2004). Códigos éticos para el mundo empresarial. Madrid: Trotta. ISBN: 9788481647068

Lozano-Aguilar, José-Félix (2007). "Códigos éticos y auditorías éticas". Veritas. Revista de filosofía y teología, v. 2, n. 17, pp. 225-251.

https://dialnet.unirioja.es/descarga/articulo/2471548.pdf

Miceli, Marcia P.; Near, Janet P.; Dworkin, Terry M. (2013). Whistle-blowing in organizations. New York: Routledge. ISBN: 9781136675713

Monasterio-Astobiza, Aníbal (2017). "Ética algorítmica: Implicaciones éticas de una sociedad cada vez más gobernada por algoritmos". Dilemata. Revista internacional de éticas aplicadas, n. 24, pp. 185-217.

https://goo.gl/gZBH8p

Nader, Ralph; Petkas, Peter; Blackwell, Kate (eds.) (1972). Whistle Blowing. USA: Bantam.

Post, Linda F.; Blustein, Jeffrey (2015). Handbook for health care ethics committees ( $2^{\text {nd }}$ ed.). Baltimore, Meriland: John Hopkins University Press. ISBN: 9781421416571

Real Academia Española (2014). "Monitorizar". Diccionario de la Lengua Española (23a edición). RAE: Madrid.
http://dle.rae.es/?id=PehHKV2

Reitaku University (1999). A guidance document for the implementation of the ethics compliance standard 2000 (ECS2000 V1.2). Reitaku University Business Ethics \& Compliance Research.

https://goo.gl/nR7ZQp

Rodríguez-Córdoba, María-Pilar; Castaño-Vélez, Carlos-Felipe; Osorio-Gómez, Valentina; Zuluaga-Arcila, Héctor-Felipe; Duque-Uribe, Verónica (2006). “La auditoría ética: herramienta para fortalecer la integridad del carácter organizacional". Innovar. Revista de ciencias administrativas y sociales, v. 16, n. 27 , pp. 25-46.

http://www.redalyc.org/articulo.oa?id $=81802703$

Rodríguez-Vaamonde, Sergio; Torre-Bastida, Ana-Isabel; Garrote, Estibaliz (2014). "Tecnologías big data para análisis y recuperación de imágenes web". El profesional de la información, v. 23, n. 6, pp. 567-574.

https://doi.org/10.3145/epi.2014.nov.02

Serrano-Cobos, Jorge (2014). "Big data y analítica web. Estudiar las corrientes y pescar en un océano de datos". El profesional de la información, v. 23, n. 6, pp. 561-565.

https://doi.org/10.3145/epi.2014.nov.01

Serrano-Puche, Javier (2012). "Herramientas web para la medición de la influencia digital: análisis de Klout y Peerlndex". El profesional de la información, v. 21, n. 3, pp. 298-303. https://doi.org/10.3145/epi.2012.may.11

Standards Australia International Committee (2001). Australian Standard AS8000. Sydney: SAIC.

Suárez-Gonzalo, Sara (2017). "Big social data: límites del modelo notice and choice para la protección de la privacidad". El profesional de la información, v. 26, n. 2, pp. 283292.

https://doi.org/10.3145/epi.2017.mar.15

Tascón, Mario; Coullaut, Arantza (2016). Big data y el internet de las cosas. Qué hay detrás y cómo nos va a cambiar. Madrid: Catarata. ISBN: 9788490970744

United Nations (1987). Our common future: Brundtland report. UN Documents: United Nations Web Side. http://www.un-documents.net/ocf-cf.htm

United Nations (2011). Guiding principles on business and human rights. New York \& Genova: United Nations. http://www.ohchr.org/Documents/Publications/GuidingPrinciplesBusinessHR_EN.pdf

United Nations (2015). Resolution adopted by the General Assembly on 25 September 2015.

http://www.un.org/ga/search/view_doc.asp?symbol=A/ $R E S / 70 / 1$ \& Lang $=E$

Vandekerckhove, Win (2006). Whistleblowing and organizational social responsibility: A global assessment, Farnham (UK). Ashgate: Publishing Ltd. ISBN: 9780754647508

Yager, Ronald R.; Pascual-Espada, Jordán (eds.) (2017). New advances in the internet of things. Cham: Springer. ISBN: 978 3319581903

https://doi.org/10.1007/978-3-319-58190-3 